\section{Giant hypothalamic hamartoma associated with an intracranial cyst in a newborn}

\author{
Joo Yeon Lee', Hye-Kyung Yoon², Shin Kwang Khang ${ }^{3}$ \\ 'Department of Radiology and Research Institute of Radiology, University of Ulsan College \\ of Medicine, Seoul; ${ }^{2}$ Department of Radiology, Kangwon National University Hospital, \\ Chuncheon; ${ }^{3}$ Department of Pathology, University of Ulsan College of Medicine, Seoul, Korea
}

We report the case of a giant hypothalamic hamartoma with a large intracranial cyst in a neonate. On ultrasonography, the lesion presented as a lobulated, mass-like lesion with similar echogenicity to the adjacent brain parenchyma, located anterior to the underdeveloped and compressed left temporal lobe, and presenting as an intracranial cyst in the left cerebral convexity without definite internal echogenicity or septa. The presence of a hypothalamic hamartoma and intracranial neurenteric cyst were confirmed by surgical biopsy. The association of a giant hypothalamic hamartoma and a neurenteric cyst is rare. Due to the rarity of this association, the large size of the intracranial cyst, and the resulting distortion in the regional anatomy, the diagnosis of the solid mass was not made correctly on prenatal high-resolution ultrasonography.

Keywords: Hypothalamic hamartomas; Infant, newborn; Central nervous system cysts;

Ultrasonography; Magnetic resonance imaging

\section{Introduction}

Hypothalamic hamartomas are rare, non-neoplastic, congenital malformations of the posterior hypothalamus that consist of ectopic neural tissue and rarely exceed $2 \mathrm{~cm}$ in diameter. When they exceed 30-40 mm in diameter, they are defined as giant hypothalamic hamartomas, and it is unclear whether the extent of a hypothalamic hamartoma affects its manifestation. To the best of our knowledge, their radiologic appearance with respect to changes in adjacent structures has not been well studied. A search of the MEDLINE and PubMed databases identified only nine articles describing 11 patients, but those studies mostly focused on the clinical features and histopathological findings of giant hypothalamic hamartomas [1]. Moreover, the association of giant hypothalamic hamartomas with intracranial cysts has not been frequently reported, and a search of the PubMed database only identified a few cases of coexisting arachnoid cysts $[1,2]$. Due to the rarity of the association of giant hypothalamic hamartomas with other intracranial lesions, the presence of intracranial cysts might lead radiologists to overlook this soft tissue mass, especially when using low-resolution diagnostic imaging, such as prenatal ultrasonography and fetal magnetic resonance (MR) imaging. The goal of this study was to present the rare case of a giant hypothalamic hamartoma in association with a non-

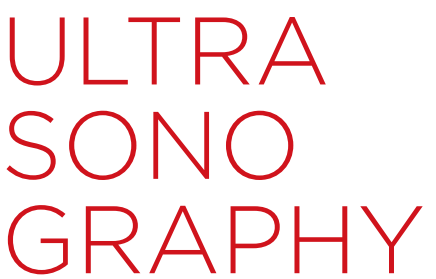

\section{CASE REPORT}

http://dx.doi.org/10.14366/usg.15080 pISSN: 2288-5919 - elSSN: 2288-5943 Ultrasonography 2016;35:353-358

Received: December 2, 2015

Revised: March 22, 2016

Accepted: March 23, 2016

Correspondence to: Hye-Kyung Yoon, MD, PhD, Department of Radiology, Kangwon National University Hospital, 156 Baengnyeongro, Chuncheon 24289, Korea

Tel. +82-33-258-9024

Fax. +82-33-258-9351

E-mail: hk2005.yoon@gmail.com
This is an Open Access article distributed under the terms of the Creative Commons Attribution NonCommercial License (http://creativecommons.org/ licenses/by-nc/3.0/) which permits unrestricted noncommercial use, distribution, and reproduction in any medium, provided the original work is properly cited.

Copyright (C) 2016 Korean Society of Ultrasound in Medicine (KSUM)

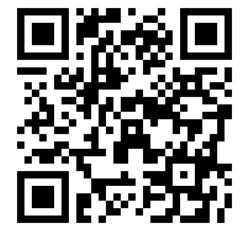

How to cite this article: Lee JY, Yoon HK, Khang SK. Giant hypothalamic hamartoma associated with an intracranial cyst in a newborn. Ultrasonography. 2016 Oct;35(4):353-358. 
arachnoid, neurenteric cyst in a newborn patient and to describe the MR features of the giant hypothalamic hamartoma.

\section{Case Report}

A 38-year-old gravida 1, para 1 female was referred to our tertiary referral center for the evaluation of an intracranial cyst demonstrated by high-resolution fetal ultrasonography at the gestational age of 25

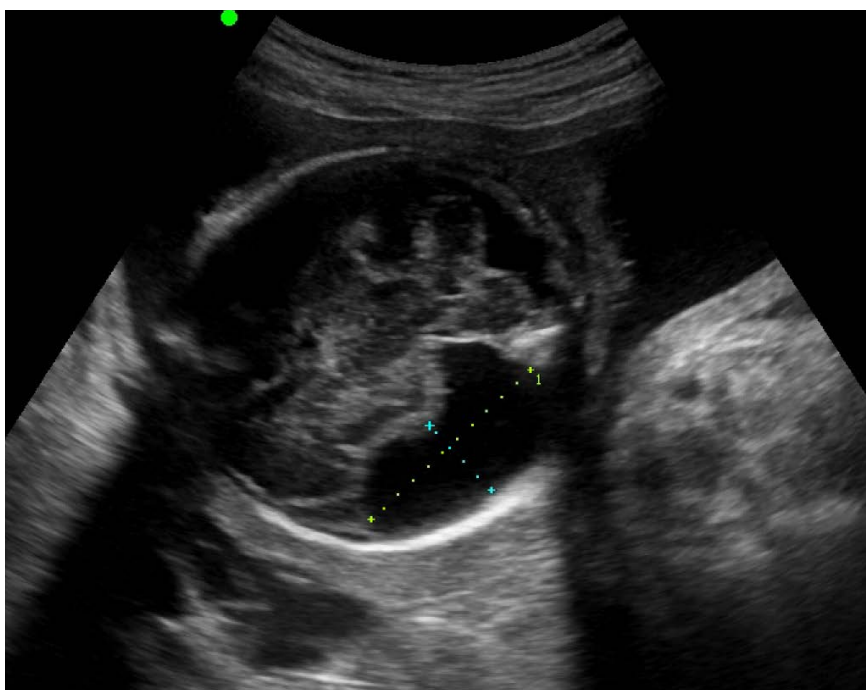

Fig. 1. Prenatal high-resolution ultrasonography at the gestational age of 28 weeks. Sonogram demonstrates a $42 \times 17-\mathrm{mm}$ intracranial cyst (dotted lines) in the left cerebral convexity without definite internal echogenicity or septa.

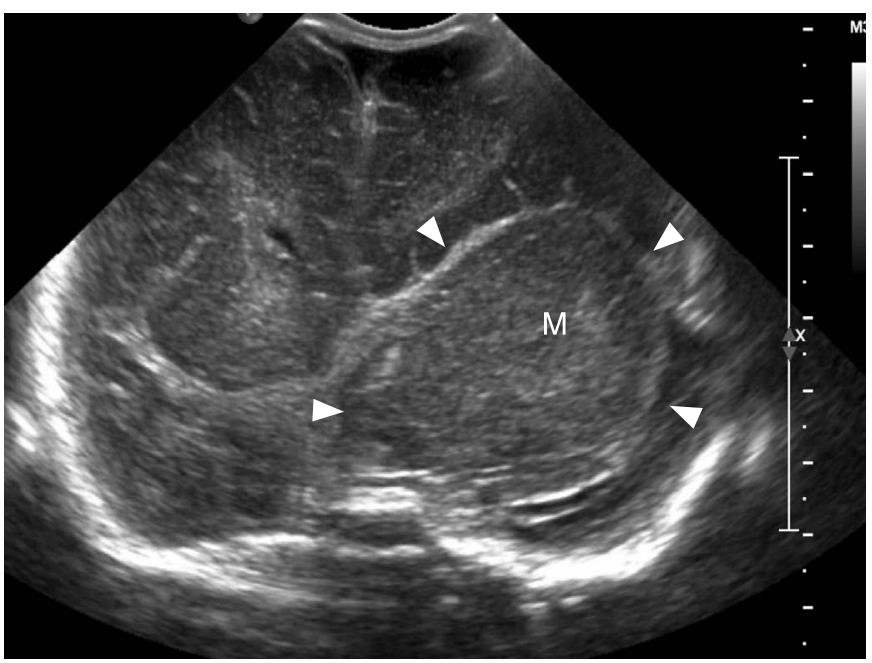

A weeks. Prenatal high-resolution ultrasonography at the gestational age of 28 weeks revealed an intracranial extra-axial cystic lesion at the left cerebral convexity with no internal echogenicity (Fig. 1), which was presumably an arachnoid cyst. Fetal cleft lip and palate were also noted in the ultrasonographic imaging. The patient was delivered at 36 weeks by vaginal delivery after augmentation with synthetic oxytocin. The male newborn was 2,892 $\mathrm{g}$ and hypotonic, but otherwise neurologically intact, with an Apgar score of 8 at 1 minute and 9 at 5 minutes. A physical examination revealed widened sutures with an enlarged head circumference $(36.5 \mathrm{~cm}$, 97th percentile), hypotonia, hypertelorism, and cleft palate and lip. The remainder of the examination was unremarkable. Postnatal ultrasonography demonstrated a lobulated mass-like lesion $(51 \times 35$ $\mathrm{mm}$ ) with similar echogenicity to the adjacent brain parenchyma, located anterior to the underdeveloped and compressed left temporal lobe, and an intracranial cyst in the left cerebral convexity with a diameter of $56 \times 22 \mathrm{~mm}$ (Fig. 2).

MR imaging at 3.0 T using high-resolution and gadoliniumenhanced T1-weighted sequences with standard T2-weighted sequences was performed on postnatal day 4 . MR imaging demonstrated a large $(60 \times 30 \times 43 \mathrm{~mm})$, lobulated left frontal solid lesion with isointense signals relative to the cortex on all pulse sequences (Fig. $3 \mathrm{~A}-\mathrm{C}$ ) and no contrast enhancement after gadolinium administration (Fig. 3D). It also contained areas with slightly high T1 signal intensity (Fig. 3B) which were considered to be a manifestation of accelerated myelination. Due to the distortion in regional anatomy and underdevelopment of the normal brain

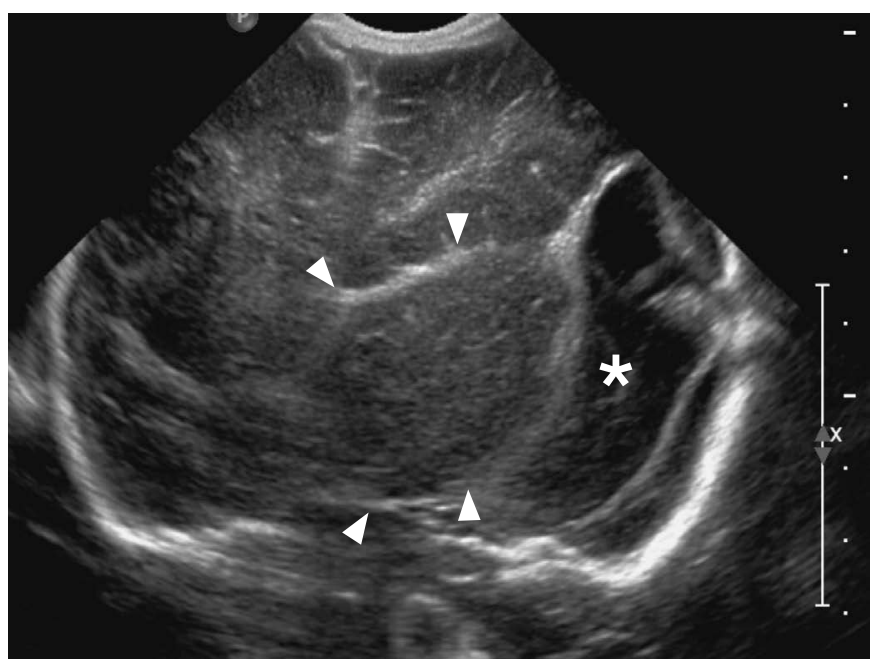

B

Fig. 2. Postnatal ultrasonography after delivery at the gestational age of 36 weeks.

A. Sonogram reveals a $51 \times 35-\mathrm{mm}$ lobulated, mass-like lesion (arrowheads) with similar echogenicity to the adjacent brain parenchyma, located anterior to the left underdeveloped and compressed temporal lobe. B. A 56×22-mm intracranial cyst (asterisk) in the left cerebral convexity is also depicted near the mass (arrowheads) on different sections of the sonograms. $M$, mass. 
resulting from the large solid mass in the frontotemporal area, a thorough evaluation was required to demonstrate the contiguity of this gyriform laminated lesion with the hypothalamus (Fig. 3C). MR spectroscopy demonstrated a similar chemical composition between the dysplastic brain tissues of the giant hypothalamic hamartoma and the normal-appearing white matter (Fig. 4). MR imaging also revealed an extremely large $(73 \times 41 \times 65 \mathrm{~mm})$ extraaxial fluid collection along the left frontoparietal convexity, which was also demonstrated on the prenatal ultrasonography. The cystic lesion was isointense relative to the cerebrospinal fluid in

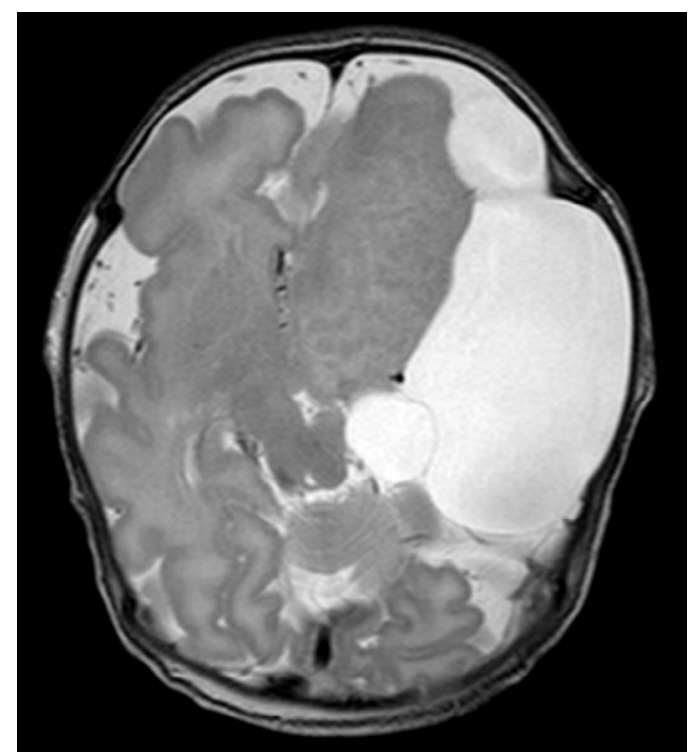

A

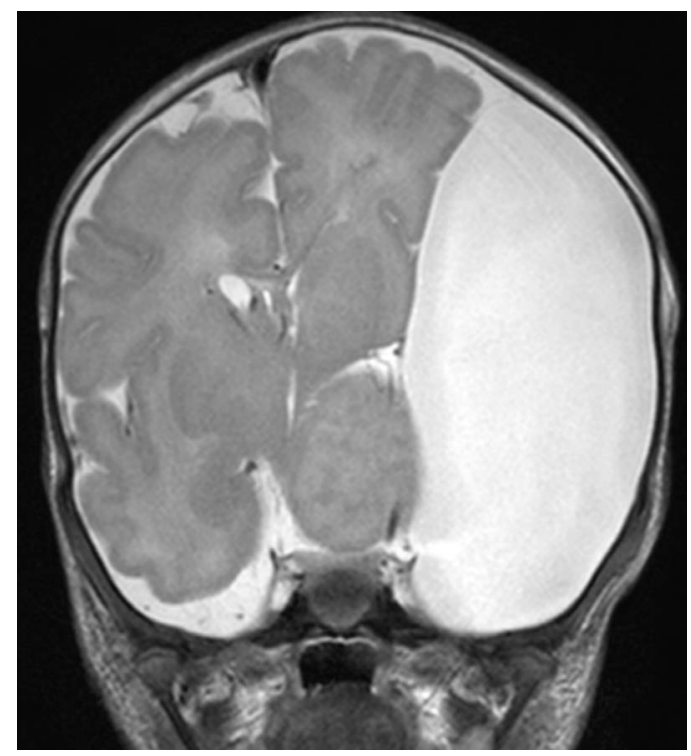

C

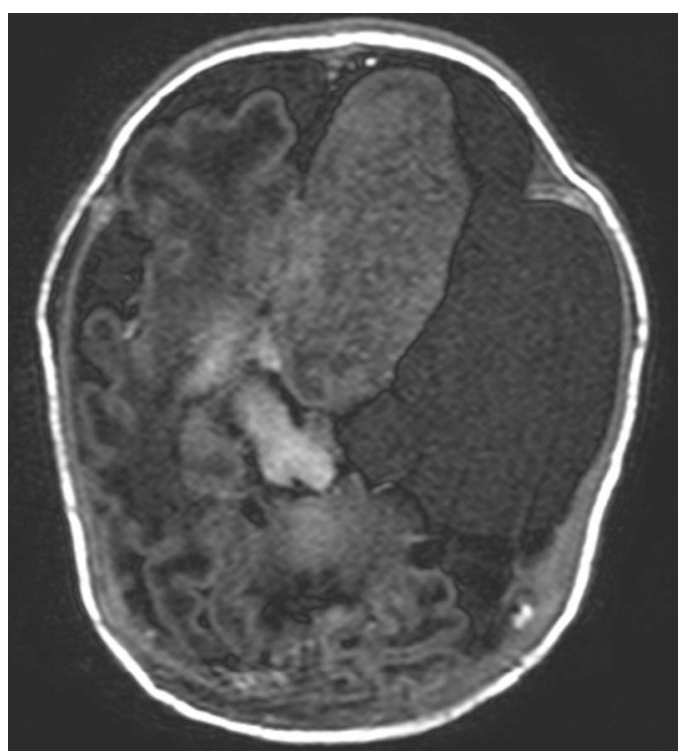

B

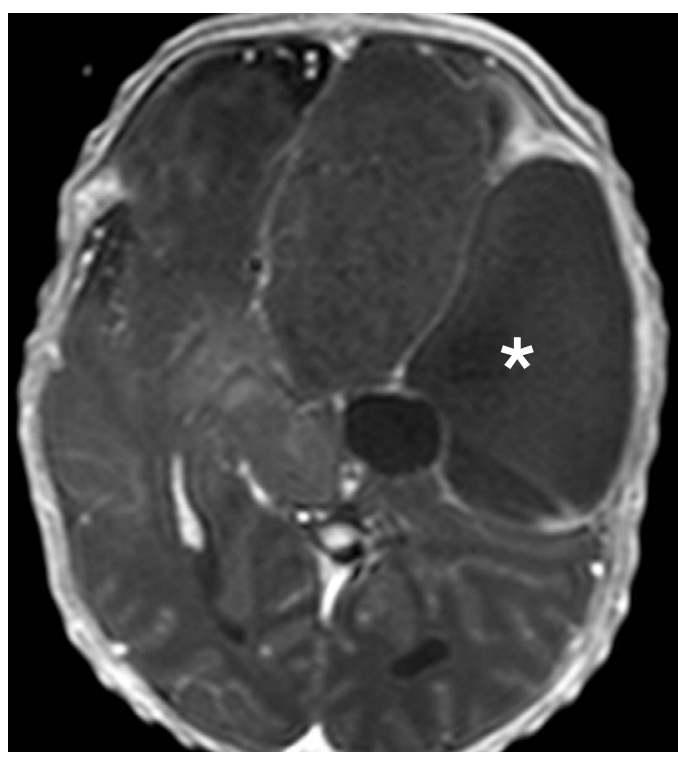

D

Fig. 3. Brain magnetic resonance (MR) imaging obtained on day 4 after delivery.

A-C. MR images demonstrate a left frontal solid mass of mixed signal intensity (, , T2-weighted images; B, T1-weighted images) with great similarity to the adjacent brain parenchyma and its contiguity with the contralateral hypothalamus, which is well visualized on the coronal T2-weighted image (C). D. Post-contrast MR image obtained 1 week later reveals a lack of enhancement in the mass on contrast enhanced, fat-saturated T1-weighted images. A huge $(73 \times 41 \times 65 \mathrm{~mm})$, extra-axial fluid collection (asterisk) along the left frontoparietal convexity corresponds to the lesion depicted on prenatal ultrasonography. Cystic lesion showed a similar signal intensity to the cerebrospinal fluid in all imaging sequences. 
all image sequences, but with suspiciously T1-hyperintense lesions in the dependent portion of the cystic locules. At the time of the presumptive diagnosis, it was assumed to be an intracystic flow artifact, considering the size of the cystic lesion, and the presumptive diagnosis was an arachnoid cyst. Surgery was performed to decompress the cystic lesion through fenestration while biopsy of the cystic wall and the solid mass were carried out.

The histologic evaluation of the cyst wall revealed single or stratified cuboidal or columnar epithelial lining instead of delicate fibrous connective tissue lined by meningothelial cells, and it was diagnosed as an intracranial neurenteric cyst. A pathologic specimen of the solid mass revealed aggregated small cells, which were not reactive to antibodies for glial fibrillary acidic protein or neuronal nuclear antigen. The background tissue expressed synaptophysin, but only few myelinated axons were observed on a Luxol fast blue-stained slide. Only a few cell processes were reactive to

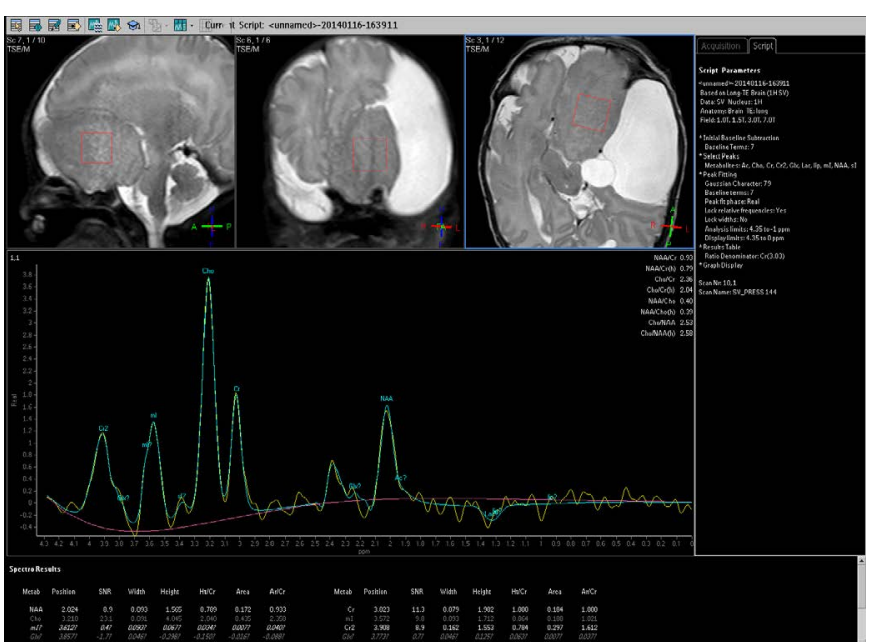

A

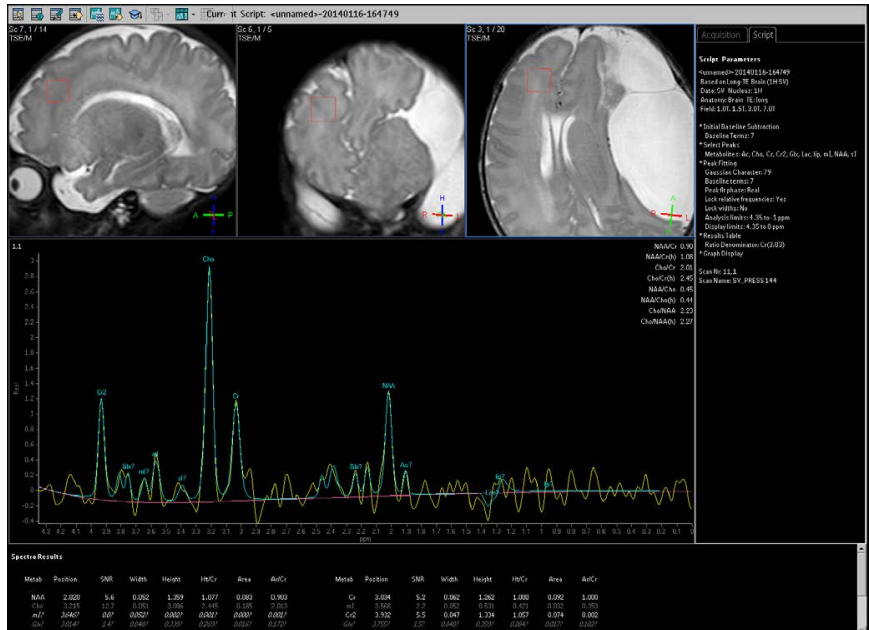

B

Fig. 4. Magnetic resonance (MR) spectroscopy of the brain tissue and the mass lesion.

A, B. Giant hypothalamic hamartoma (A) and the white matter of the dysplastic brain (B) showed a similar chemical composition to each other on MR spectroscopic analysis.

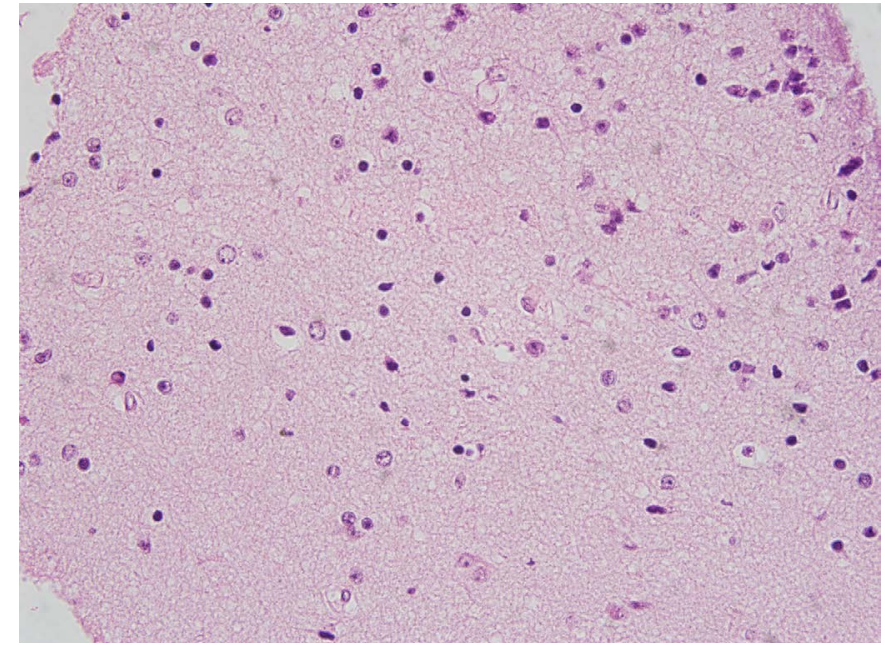

A

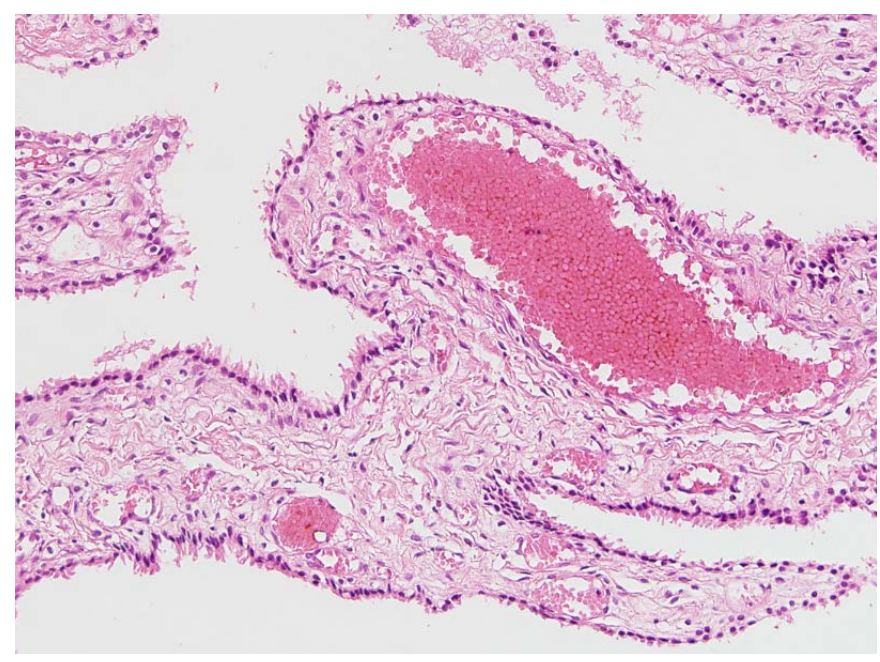

B

Fig. 5. Microscopic images of the tumor.

A. Solid mass demonstrated an aggregation of small cells that were not reactive to antibodies for glial fibrillary acidic protein and neuronal nuclear antigen. No cellular atypia was noted. All these features are consistent with a hypothalamic hamartoma (H\&E, $\times 400)$. B. Cyst wall exhibited single or stratified cuboidal or columnar epithelial lining instead of the delicate fibrous connective tissue lined by meningothelial cells associated with intracranial neurenteric cysts (H\&E, $\times 100)$. 
phosphorylated neurofilament. No cellular atypia was noted. All these features are consistent with a hypothalamic hamartoma (Fig. 5). The further development of the patient was quite satisfying, although he exhibited hypotonia and intermittent jitteriness of both lower legs without significant delays in developmental milestones.

\section{Discussion}

With a benign and congenital nature, hypothalamic hamartomas are non-neoplastic, rare $(1: 200,000)$ lesions formed by ectopic neural tissue, and are commonly associated with gelastic seizures and precocious puberty. Patients with giant hypothalamic hamartomas frequently have seizures $(63.6 \%)$, with the majority of the seizures belonging to the gelastic type and a prevalence similar to that observed in patients with non-giant hypothalamic hamartomas [3]. The seizures might be caused by the possible presence of associated cortical dysgenesis, although mechanical compression of the hypothalamus, including the mammillary bodies, is also considered to be a significant factor in the emergence of seizures.

Hypothalamic hamartomas are also commonly associated with precocious puberty $(63 \%)$, and are the most common identifiable neurological cause of this phenomenon, followed by hypothalamic and chiasmatic gliomas, germ cell tumors, arachnoid cysts, and isolated hydrocephalus. The theory for the pathogenesis of precocious puberty is that the hamartoma may be directly involved in neurosecretion or result in a physical perturbation of inhibitory pathways. In contrast to the non-giant hypothalamic hamartomas, giant hypothalamic hamartomas were associated with precocious puberty only in $36.3 \%$ of the patients. This report describes the first case of a giant hypothalamic hamartoma associated with a non-arachnoid, neurenteric intracranial cyst. As hypothalamic hamartomas have been attributed to abnormal splitting of the notochord, which could result in other known associated congenital anomalies of the craniofacial and central nerve system, hypothalamic hamartomas may be part of the wide spectrum of associated craniofacial developmental abnormalities, including hypertelorism, cleft lip and palate, basal encephaloceles, septo-optic dysplasia, callosal agenesis, and holoprosencephaly [4]. In our case, a congenital hypothalamic mass was also identified in association with the craniofacial anomalies of hypertelorism and cleft lip and palate.

Previously, the coexistence of an arachnoid cyst and a hypothalamic hamartoma has been reported in a few cases, and has been explained through two theories $[1,2,5]$. One relates this phenomenon to the interruption of the normal development of the subarachnoid space, which begins with the expansion of the intracellular space as the meninx primitiva degenerates at 14 weeks, potentially leading to the formation of an arachnoid cyst [6]. The other theory is that arachnoid or meningeal tissue could be within the hamartoma from the beginning, contributing to the formation of an associated arachnoid cyst $[7,8]$. However, other intracranial cystic lesions have not been well studied and remain poorly understood. The sonographic findings associated with this condition have likewise not yet been well studied. In our case, the lesion presented as a lobulated mass-like lesion with similar echogenicity to the adjacent brain parenchyma. MR imaging has been proven to be the modality of choice for the diagnosis of hypothalamic hamartomas. They are usually ovoid lesions located close to the posterior hypothalamus, tuber cinereum, or mammillary bodies, and may be sessile or peduncluated and small, rarely exceeding $2 \mathrm{~cm}$ in diameter. The reported atypical features of hypothalamic hamartomas include giant hamartomas, lipomatous content, cystic necrosis, and prominent $\mathrm{T} 2$ prolongation [9], with giant hypothalamic hamartomas defined as those with a diameter exceeding $30-40 \mathrm{~mm}$. A search of the PubMed database for the keywords "giant," "large," or "voluminous" hypothalamic hamartomas in the titles identified only nine articles describing 11 patients. These studies mostly dealt with the clinical presentation, histopathology, and clinical course of the hamartomas. The reported imaging findings include isointensity relative to the adjacent gray matter without contrast enhancement in all 11 cases, and three case out of the 11 included cystic changes [3,10-12].

The presence of large intracranial cysts associated with a hamartoma can lead to significant alterations in the regional anatomy and an incorrect preoperative diagnosis. Although the association with intracranial cysts is rare, the presumptive diagnosis of hamartoma should be made when the MR imaging features of the solid component are typical for a hypothalamic hamartoma and exhibit continuity with the remaining hypothalamus. These features should be evaluated in any patient presenting with a huge brain parenchymal mass, which is too large for its origin to be clearly demonstrated due to distortions in the regional anatomy. We suggest that isointense lamellate gyriform masses in fetal and postnatal MR imaging should be carefully evaluated for continuity with the adjacent structures, especially with the hypothalamus, as well as for their signal intensity relative to the adjacent cortex.

ORCID: Joo Yeon Lee: http://orcid.org/0000-0001-8964-7107; Hye-Kyung Yoon: http://orcid.org/0000-0001-7547-0320; Shin Kwang Khang: http://orcid.org/00000003-3707-6802

\section{Conflict of Interest}

No potential conflict of interest relevant to this article was reported. 


\section{References}

1. Booth TN, Timmons C, Shapiro K, Rollins NK. Pre- and postnatal MR imaging of hypothalamic hamartomas associated with arachnoid cysts. AJNR Am J Neuroradiol 2004;25:1283-1285.

2. Elliott RE, Tanweer O, Rubin BA, Koslow M, Mikolaenko I, Wisoff JH. Suprasellar hamartoma and arachnoid cyst. World Neurosurg 2013;80:e401-e407.

3. Alves C, Barbosa V, Machado M. Giant hypothalamic hamartoma: case report and literature review. Childs Nerv Syst 2013;29:513516.

4. Kollias SS, Ball WS, Prenger EC. Review of the embryologic development of the pituitary gland and report of a case of hypophyseal duplication detected by MRI. Neuroradiology 1995;37:3-12.

5. Isaka T, Nakatani S, Yoshimine T, Akai F, Taneda M. Asymptomatic hypothalamic hamartoma associated with an arachnoid cyst--case report. Neurol Med Chir (Tokyo) 1996;36:725-728.

6. McLone DG. The subarachnoid space: a review. Childs Brain 1980;6:113-130.
7. Goda M, Tashima A, Isono M, Hori S, Kimba Y. A case of hypothalamic hamartoma associated with arachnoid cyst. Childs Nerv Syst 1999;15:490-492.

8. Nishio S, Morioka T, Hamada Y, Kuromaru R, Fukui M. Hypothalamic hamartoma associated with an arachnoid cyst. J Clin Neurosci 2001;8:46-48.

9. Tonami H, Higashi K, Okamoto K, Akai T, lizuka H, Nojima T, et al. Report of changing signal intensity on follow-up MRI in a case of hypothalamic hamartoma. J Comput Assist Tomogr 2001;25:130132.

10. Aran E, Pereira J, Castro L, Vaz R. Voluminous hypothalamic hamartoma in a 5-month-old boy: Epilepsy and surgery. Neurocirugia (Astur) 2004;15:294-297.

11. Dorfer C, Kasprian G, Muhlebner A, Czech T. Giant solidcystic hypothalamic hamartoma. Case report. Neurosurg Focus 2011;30:E7.

12. Miranda P, Esparza J, Cabrera A, Hinojosa J. Giant hypothalamic hamartoma operated through subfrontal approach with orbitary rim osteotomy. Pediatr Neurosurg 2006;42:254-257. 